\title{
MODELLE DER TRADITIONSBILDUNG IN KILL BILL: VERRAT, MORD, RACHE
}

\author{
OLIVER KOHNS \\ »Derjenige, der arbeiten will, gebiert seinen eigenen Vater. « \\ (Søren Kierkegaard: Furcht und Zittern) \\ »The apple doesn't fall far from the tree.«
}

\section{Verrat}

Solange Nachahmung grundsätzlich positiv bewertet wird, stellt sie noch kein prinzipielles philosophisches Problem dar. Das Problem der Nachahmung und ihrer Ausformungen - Mimesis, Nachfolge, Beeinflussung - ist unter diesem Vorzeichen lediglich: Wer wird nachgeahmt und wie wird nachgeahmt? ${ }^{2}$ Die Situation ändert sich, sobald Nachahmung grundsätzlich negativ bewertet wird: Wie kann man jemandem nachfolgen, der jede Form von Nachfolge ablehnt, um selbst zu denken und selbst zu >schaffen<? Und umgekehrt: Auf welche Nachfolger darf ein Autor hoffen, zu dessen Prinzipien zuallererst die Absage an jede Form des blinden Glaubens an Überlieferung und Tradition und also auch an >Nachfolge< gehört?

Um das Problem der Nachfolge kreist einer der Aphorismen aus Nietzsches Fröhlicher Wissenschaft.

1 Søren Kierkegaard: Furcht und Zittern. Dialektische Lyrik von Johannes de Silentio. Übers. von Liselotte Richter, Hamburg: Europäische Verlagsanstalt 1998, S. 23.

2 Vgl. Philippe Lacoue-Labarthe: "Hölderlin und die Griechen [1979]«, in: ders.: Die Nachahmung der Modernen. Typographien II. Übers. von Thomas Schestag. Basel, Weil am Rhein, Wien: Engeler [0.J.], S. 71-85; René Girard: »Innovation und Wiederholung «, in: ders.: Die verkannte Stimme des Realen. Eine Theorie archaischer und moderner Mythen, Übers. von Petra Willim. München, Wien: Hanser 2005, S. 202-221. 
»Unerwünschte Jünger. - Was soll ich mit diesen beiden Jünglingen machen! rief mit Unmut ein Philosoph, welcher die Jugend >verdarb<, wie Sokrates sie einst verdorben hat - es sind mir vollkommen unwillkommene Schüler. Der da kann nicht Nein sagen und Jener sagt zu Allem: 'Halb und halb<. Gesetzt, sie ergriffen meine Lehre, so würde der Erstere zu viel leiden, denn meine Denkweise erfordert eine kriegerische Seele, ein Wehethun-Wollen, eine Lust am Neinsagen, eine harte Haut, - er würde an offenen und inneren Wunden dahin siechen. Und der Andere wird sich aus jeder Sache, die er vertritt, eine Mittelmäßigkeit zurecht machen und sie dergestalt zur Mittelmässigkeit machen, - einen solchen Jünger wünsche ich meinem Feinde. « ${ }^{3}$

Der Aphorismus umschreibt präzise das Problem einer Nachfolge desjenigen, der jede Nachfolge kategorisch ablehnt. Um die `Lehre , des Philosophen zu >ergreifen`, müssen die beiden `Jünger« vor allem lernen, keine Jünger zu sein. Der wahre Nachfolger des Philosophen wäre derjenige, der kein Nachfolger ist. Solange ein Schüler >nicht Nein sagen k kann, ist er kein würdiger Schüler dieses Philosophen und bleibt >vollkommen unwillkommen<. Nietzsches Formulierung, ein solcher Jünger würde >zu viel leiden $\iota$, schließt an die ältere Bedeutung von >leiden, erleiden ‘ als Ausübung einer Passivität an; sie gewinnt in Verbindung mit der Forderung Nietzsches nach einer >kriegerischen Seele und einer >harten Haut jedoch zugleich eine martialische Wörtlichkeit.

Die Lehre der Philosophie erfordert, wie es in einem anderen Aphorismus heißt, »das erlangte gute Gewissen bei der Feindseligkeit gegen das Gewohnte, Ueberlieferte, Geheiligte «. ${ }^{4}$ Denken wird damit zu einer kriegerischen Auseinandersetzung gegen jede Form der Tradition und Nachahmung. Diese Feindseligkeit muss sich aber auch gegen jede Tradition und Nachahmung der >Feindseligkeit gegen das Gewohnte richten: Der Philosoph mit >kriegerischer Seele kann letztlich weder Vorbilder noch Nachfolger haben, weder Meister noch Jünger. »Man vergilt einem Lehrer schlecht, wenn man immer nur der Schüler bleibt $\aleph^{5}$, heißt es in Also sprach Zarathustra.

Doch kann man jemals ınein sagen zu einer solche Philosophie des \Neinsagens`? Ebenso, wie man die Nachfolge durch Zustimmung zur Lehre des Meisters notwendigerweise verfehlen muss, ist jede Ableh-

3 Friedrich Nietzsche: Die Fröhliche Wissenschaft, 32, in: Kritische Studienausgabe in 15 Einzelbänden. Hrsg. von Giorgio Colli und Mazzino Montinari. München: Dtv; Berlin: de Gruyter 1988, Bd. 3, S. 403.

4 Nietzsche: Fröhliche Wissenschaft, 297. - Kritische Studienausgabe (wie Anm. 3), Bd. 3, S. 537.

5 Nietzsche: Also sprach Zarathustra, in: Kritische Studienausgabe (wie Anm. 3), Bd. 4, S. 101. 
nung des Philosophen zugleich ein >Ergreifen< seiner Lehre, auch wenn es gerade dies nicht beabsichtigen mag. Die Jünger befinden sich mit anderen Worten in einer Situation des $>$ double bind $:^{6}$ Der Philosoph fordert von ihnen zugleich Nachfolge und Abweichung, weshalb jede Äußerung ihrer Zustimmung Abweichung bedeutet, während jedes >Neinsagen` eine Nachfolge signalisiert. Auch wenn das Wort >verderben hier ausdrücklich nur in ironisierenden Anführungszeichen erscheint, muss man zu dem Urteil kommen, dass ein solcher Philosoph in der Tat die Jugend >verdirbt

Welche Mittel gibt es, mit dieser Situation eines >double bind umzugehen? Einem Jünger des Philosophen wäre allenfalls zu raten, jederzeit einen Dolch unter seinem Gewand zu tragen, womit er den Meister eines Tages überraschen kann, um diesen von seiner Unabhängigkeit $\mathrm{zu}$ überzeugen. In einem weiteren Aphorismus aus der Fröhlichen Wissenschaft, der sich als eine Fortsetzung des zitierten 32. Aphorismus verstehen lässt, spricht Nietzsche jedenfalls von der »Fähigkeit zur Rache«:

»Fähigkeit zur Rache. - Dass Einer sich nicht vertheidigen kann und folglich auch nicht will, gereicht inm in unsern Augen noch nicht zur Schande: aber wir schätzen Den gering, der zur Rache weder das Vermögen noch den guten Willen hat, - gleichgültig ob Mann oder Weib. Würde uns ein Weib festhalten (oder wie man sagt , fesseln<) können, dem wir nicht zutrauten, dass es unter Umständen den Dolch (irgend eine Art von Dolch) gegen uns gut zu handhaben wüsste? Oder gegen sich: was in einem bestimmten Falle die empfindlichere Rache wäre (die chinesische Rache). ${ }^{7}$

Die Paradoxie der Traditionsverweigerung hat nach Nietzsche (und bereits vor ihm) zahlreiche Autoren beschäftigt. In Harold Blooms Theorie der Anxiety of Influence wird die Angst, die ein übermächtiger Vorgänger einem Schriftsteller bereiten kann, nur durch die Sorge übertroffen, dass auch dieser Vorgänger sich wiederum gegen seine Vorgänger zu wehren hatte und dass folglich jede Form der Rebellion gegen die Tradition ein zutiefst traditioneller Akt ist. ${ }^{8}$ Bereits Lichtenberg no-

6 Vgl. Gregory Bateson: Vorstudien zu einer Theorie der Schizophrenie [1956], in: ders.: Ökologie des Geistes. Anthropologische, psychologische, biologische und epistemologische Perspektiven. Übers. Hans Günter Holl. 5. Aufl., Frankfurt a.M.: Suhrkamp 1994, S. 270-301.

7 Nietzsche: Fröhliche Wissenschaft, 69. - Kritische Studienausgabe (wie Anm. 3), Bd. 3, S. $427 f$.

8 Vgl. Harold Bloom: Einflußangst. Eine Theorie der Dichtung. Übers. von Angelika Schweikhart, Frankfurt a.M.: Stroemfeld 1995 (Nexus. 4), bes. S. 64: »Mit >dichterischem Einfluß meine ich nicht die Transmission von Ideen und Bildern von früheren zu späteren Dichtern, denn das ist einfach setwas, das 
tiert das Dilemma der Jünger aus Nietzsches Aphorismus: »Grade das Gegenteil tun heißt auch nachahmen, es heißt nämlich das Gegenteil nachahmen. $"{ }^{9}$ (In dieser Paradoxie kann ohne weiteres auch eine Aporie jeder Avantgarde-Ästhetik gesehen werden.)

Insbesondere aber Quentin Tarantinos Film KILL BILL ist über weite Strecken kaum etwas anderes als eine Meditation über jenen »Fähigkeit zur Rache« betitelten Aphorismus Nietzsches. Die Braut, jene »tödlichste Frau ${ }^{10}{ }^{0}$, ist die Inkarnation des $>$ Weibes $\iota$, das jede Art von Dolch $>$ unter Umständen [...] gegen uns gut zu handhaben wüsste<. Auch ohne explizit genannt $\mathrm{zu}$ werden, ist Nietzsche in Tarantinos Film jederzeit gegenwärtig: zahlreiche Diskurse zitieren Elemente aus Nietzsches Texten oder erinnern zumindest an eine trivialisierte Version des Nietzscheanismus. Hattori Hanzos Lehre von der Mitleidslosigkeit des idealen Rächers $^{11}$ zitiert Nietzsches Invektiven gegen das Mitleid ${ }^{12}$, und Bills >Superman<-Rede mutet an wie eine überdrehte Fassung der Rede vom >Übermenschen` in Nietzsches Also sprach Zarathustra ${ }^{13}$ unter dem Einfluss von zahllosen Superman-Heften. Jenseits dieser Zitierungen und Anspielungen ist der philosophische Gehalt von KILL BILL weitaus eher in seinen Reflexionen über das Verhältnis von Rache und Nachfolgerschaft zu suchen.

KILL BILL ist ein Film über Rache; das ist offensichtlich. ${ }^{14}$ Es ist aber zugleich auch ein Film über Lehrer und Schüler, über Väter und Söhne, über Väter und Töchter und also über Nachfolge und Nachahmung. Insofern sich das Verhältnis all dieser Paarbeziehungen von Vorgänger und Nachfolger als >Feindseligkeit`, als >kriegerischeく Auseinander-

geschieht ‘, und ob eine solche Transmission bei den späteren Dichtern Angst hervorruft, ist bloß eine Frage des Temperaments und der Umstände. [...] Doch der Standpunkt eines Dichters, sein Wort, seine imaginative Identität, sein ganzes Wesen müssen für inn etwas Einzigartiges sein und bleiben, oder er wird als Dichter untergehen, selbst wenn er seine Wiedergeburt in dichterische Inkarnation bewerkstelligt hat. Aber dieser fundamentale Standpunkt ist auch der seines Vorgängers, so wie der Grundcharakter eines Menschen auch der seines Vaters ist, auch in einer Umwandlung, auch in einer Umkehrung. «

9 Georg Christoph Lichtenberg: Sudelbücher [D 604]. - Schriften und Briefe. Bd. 1-4. Hrsg. von Wolfgang Promies. München: Hanser 1968, Bd. 1, S. 321. Vgl. Bloom: Einfluss-Angst (wie Anm. 7), S. 31.

10 KILL BILL: Volume 2, 01:47:27.

11 Vgl. KILL BILL: Volume 1, 00:14:41.

12 »Was ist schädlicher als irgendein Laster? - Das Mitleiden der That mit allen Missrathnen und Schwachen - das Christenthum « (Nietzsche: Der Antichrist. Kritische Studienausgabe [wie Anm. 3], Bd. 6, S. 170).

13 "'Todt sind alle Götter: nun wollen wir, dass der Übermensch lebe. - diess sei einst am grossen Mittage unser letzter Wille! (Nietzsche: Also sprach Zarathustra. - Kritische Studienausgabe [wie Anm. 3], Bd. 4, S. 102).

14 Vgl. Robert Fischer: »Kill Bill«, in: Robert Fischer/Peter Körte/Georg Seeßlen (Hg.), Quentin Tarantino, Berlin: Bertz + Fischer 2004, S. 197-234, hier: S. 217. 
setzung oder als >Racheく bestimmt, gehören beide Themenfelder in Tarantinos Film notwendig zusammen. Dieser Zusammenhang kann erklärt und analysiert werden mit Blick auf die Paradoxie der Traditionsverweigerung, wie sie in Nietzsches Fröhlicher Wissenschaft entfaltet wird.

Aus der Lektüre des Films KILL BILL ergeben sich drei Thesen über den Zusammenhang der Gewalttätigkeit mit den Problemen der Traditionsbildung. Erstens: Innerhalb einer Ökonomie der Rache ist die Ermordung eines Vorgängers stets die wahre Aneignung seiner Lehre (dies folgt aus der Paradoxie der Traditionsverweigerung). Zweitens: Wenn die Ermordung des Lehrers die höchste Stufe des Lernens ist, kann das Vorbild niemals wirklich verraten werden (denn jeder Verrat kann nur den Verrat des Meisters an seinem Vorgänger wiederholen und stellt daher eine vollkommene Aneignung der Position des Lehrers dar). Drittens: Die Sequenz der Rache kann prinzipiell kein Ende nehmen (denn jeder Jünger wird anderen Nachfolgern zum Vorgänger werden). Diese drei Thesen sollen im Folgenden näher entfaltet werden.

\section{Mord}

Das allgemeinste Schema der Rache ebenso wie der Nachfolge durch Rache erzählt die Vorgeschichte O-Ren Ishiis, die von der Stimme der Braut als Voice-Over über eine Zeichentricksequenz berichtet wird. Der Zeichentrickeinschub ist im Stil eines japanischen >Mangas`gehalten, was nicht nur Japan als den Ort der Handlung unterstreicht, sondern auch die extreme Gewalt und eine recht pornographische Sequenz darstellbar macht. Erzählt wird, wie »Japan's most ruthless Yakuza boss, Boss Matsumoto ${ }^{15}$ die Eltern der neunjährigen O-Ren Ishii brutal ermorden lässt, während diese sich zitternd unter dem Bett verstecken kann. Doch der Ausbruch der Gewalt hat auch mit dem abschließenden Niederbrennen ihres Elternhauses vor den Augen O-Ren Ishiis kein Ende: »She swore revenge... luckily for her, Boss Matsumoto was a pedophile. ${ }^{16}$ Nur zwei Jahre später erhält sie ihre Rache: Man sieht, wie die elfjährige O-Ren Ishii in der Uniform eines Schulmädchens und in eindeutiger Position auf dem Boss der Yakuza sitzt und ihm in einem überraschenden Moment ein langes Schwert in den Leib rammt.

Die Handlung der Rache eröffnet hier ein Feld der Ähnlichkeiten. Die Geste des >Durchbohrens` erinnert an den Tod der Mutter O-Ren Ishiis, die gleichfalls auf dem Bett liegend von einem Schwert durchstoßen 
wurde. Die Fontäne des in alle Richtungen ausströmenden Blutes erinnert zudem an den Mord an ihrem Vater. O-Ren Ishii spricht die Frage der Ähnlichkeit explizit an, indem sie den röchelnden und sterbenden Yakuza-Boss auf ihre Ähnlichkeit zu ihrer ermordeten Mutter aufmerksam macht: »Sieh mich an, Matsumoto. / Präg dir mein Gesicht gut ein. / Sieh mir in die Augen. / Sieh auf meinen Mund. / Komm ich dir bekannt vor? Sehe ich jemandem ähnlich, den du ermordet hast? « ${ }^{17}$ Wie die Flammen des brennenden Elterhauses sich in den Augen der jungen O-Ren gespiegelt haben, so zeigt nun ein >Close Up $<$ in den Augen des sterbenden Matsumoto das Gesicht O-Rens: Eine gespiegelte Spiegelung. Die gelungene Rache ähnelt so der Tat, die sie rächt: Sie wiederholt sie in umgekehrter Besetzung, indem die Tochter den Mörder ihrer Mutter in deren Namen und im Zeichen ihrer Ähnlichkeit ermordet. Rache vergilt >Gleiches mit Gleichem`. (Das Feld der Ähnlichkeiten spielt auch in dem Rachefeldzug der Braut eine wichtige Rolle: nahezu jeder Moment des Wiedersehens mit einem der Beteiligten an dem Massaker in El Paso wird durch eine markante Tonfolge begleitet, die Wiedererkennen und Erinnerung bezeichnet. Damit ergibt sich auch hier eine Korrespondenz zwischen ihrer Rache und der motivierenden Tat, aber diese Beziehung wird in das Gedächtnis der Braut verlegt.)

Paradoxerweise entwickelt die Rache ihre Kraft der Ähnlichkeit und Verähnlichung aber auch in eine andere Richtung: O-Ren Ishii wird im Anschluss an die Ermordung Matsumotos zum Profi-Killer und steigt, nach einem »Shakespearian-in-magnitude power struggle with the other Yakuza clans $«{ }^{18}$ zum $»$ Boss der Bosse $«{ }^{19}$ in der Tokioter Unterwelt auf. Statt den Kampf ihres Vaters gegen die Yakuza fortzusetzen, wird sie folglich zur legitimen Nachfolgerin von Boss Matsumoto. Ihr grausames Regime in der Unterwelt Tokios steht dem des Mannes, den sie ermordet hat, wohl kaum nach. Der Boss Matsumoto ist damit nicht nur der Mann, der O-Ren Ishiis Eltern ermorden ließ und den sie dafür tötete, sondern auch ihr Lehrer. Ihre weitere Karriere verläuft nach seinem Vorbild.

Die Vorgeschichte O-Ren Ishiis entfaltet damit >en miniature Drama der Traditionsverweigerung, wie Nietzsche es in seinen Aphorismen beschreibt. Wie Boss Matsumoto am eigenen Leib erfahren muss, ist auch O-Ren Ishii eine Verkörperung jenes Weibs, das einen Dolch »unter Umständen [...] gegen uns gut zu handhaben wüsste«. 


\section{Nachfolge}

Die Beziehung zwischen O-Ren Ishii und Boss Matsumoto gibt derart ein Präludium für das Verhältnis der Rächer zu ihren Lehrmeistern. In KILL BILL treten mindestens drei Figuren als >Lehrmeister` auf: zunächst Hattori Hanzo, der die Braut in die Kunst und Technik der Rache einführt, der aber auch schon Bills Lehrer war; sodann Pai Mei, der ebenfalls Bill und die Braut, aber auch Elle Driver ausbildet; und schließlich Bill selbst, der mindestens für die Braut und wiederum für Elle Driver die Rolle eines Meisters einnimmt.

Anhand der Beziehung Bills zu den anderen Figuren zeigt sich indes, dass das Verhältnis von Meister zu Schüler sehr bald noch die verschiedensten zusätzlichen Konnotationen annehmen kann. Bill ist der Lehrer und >Meister der Braut, gewiss (»Bill ist dein Meister $\aleph^{20}$, sagt Pai Mei zur Braut); er ist aber ebenso auch ihr Liebhaber und der Vater ihres Kindes. Mit einem für einen Profikiller unerwarteten Eingeständnis emotionaler Beteiligung erklärt er gegen Ende des zweiten Teils das Massaker in der Kirche zu El Paso damit, dass die Braut ihm mit seinem plötzlichen Verschwinden >das Herz gebrochen< habe: »There are consequences to breaking the heart of a murdering bastard. ${ }^{21}$ Ähnlich verdoppelt ist auch die Beziehung zwischen Bill und Elle Driver. Bill ist ebenfalls ihr Lehrmeister und Liebhaber zugleich; telephonisch gesteht er ihr seine Liebe zu ihr, als Elle am Krankenbett der Braut steht. ${ }^{22}$ Für die Braut ist Bill jedoch nicht nur Lehrer und Liebhaber, sondern auch eine Vaterfigur: Wenn er sich in der Kirche in El Paso als der Vater der Braut ausgibt, macht er nur eine Rolle explizit, die er immer wieder einnimmt. Das >Deadly Viper Assassination Squad`, das Team der Profikiller, der die Braut angehörte und das sie im Laufe des Films nach und nach auslöscht, ist Bills Erfindung, eine Geburt seines Gehirns, seine Schöpfung. Die Braut ist ebenso das Geschöpf Bills wie ihre tödlichen Schwestern Elle Driver, Vernita Green und O-Ren Ishii. (Allein Budd ist nicht der Sohn Bills, sondern sein Bruder, und entsprechend hoch ist auch die Verachtung Bills für ihn.)

»Like most men, who never knew their father, Bill collected father figures $\aleph^{23}$ behauptet die Braut - aber dieser Satz trifft auf sie genauso zu wie auf Bill. Neben Hattori Hanzo und Pai Mei ist Bill für sie die zentrale Vaterfigur - und es ist kein Widerspruch dazu, sondern eine tödliche Konsequenz, dass das >telos` der gesamten Narration auf die Tötung Bills durch ihre Hand (`Kill Bill $)$ ausgerichtet ist. Den narrativen

20 KILL BILL: VolUME 2, 00:46:03.

21 KILL BILL: VOLUME 2, 01:52:33.

22 KILL BILL: VolUMe 1, 00:23:08.

23 KILL BILL: Volume 2, 01:19:06. 
Motor des Films bildet, wie in Freuds Theorem des Ödipuskomplexes und in seiner kulturtheoretischen Abhandlung Totem und Tabu, ein Vatermord. Während der Mord am mythischen Ur-Vater in Totem und Tabu der Anfang aller Geschichte überhaupt ist ${ }^{24}$, erscheint er in KILL BILL als deren absolutes Ende, denn die Braut tötet zuerst ihre sämtlichen Geschwister mitsamt deren Anhang (die gesamte mythische Urhorde) und anschließend erst den Vater, der die Horde erfunden und in seinem Kopf geboren hat: Bill.

Auf dem Weg zu dieser Tötung ist die Braut jedoch nur erfolgreich, indem sie zu einer noch tödlicheren Version Bills selbst wird. Sie geht durch die gleiche Ausbildung (bei Pai Mei und bei Hattori Hanzo), die auch Bill durchlaufen hat, und bekämpft ihre zentrale Vaterfigur so durch weitere Vaterfiguren. Sie schlägt Bill folglich buchstäblich mit dessen eigenen Waffen (mit denen von Hattori Hanzo nämlich, die auch Bill für unbezahlbar hält) und mit dessen eigener Technik. »The apple doesn't fall far from the tree $\ll^{25}$ sagt Bill vor dem Massaker in der Kirche zu El Paso über die Fähigkeit der Braut, ihn zu >überraschen` - und macht damit, wie in einer antiken Tragödie, eine unwissende Aussage über seine eigene Zukunft und sein eigenes Ende.

Die Braut geht den gleichen Weg wie vor ihr Bill, sie überbietet ihn jedoch: Hattori Hanzo schmiedet für sie noch einmal ein Schwert, damit sie seinen ehemaligen Schüler Bill töten kann, und Pai Mei bringt nur ihr (nicht aber Bill) die >Five-Point-Palm-Exploding-Heart-Technique Durch die Tötung Bills am Ende des zweiten Teils erweist sie sich als die perfekte und vollendete Schülerin, die sie ist, und Bill selbst zögert nicht, ihr seinen Respekt auszusprechen. Ihr Rachefeldzug bedeutet an keiner Stelle einen Bruch mit Bill, sondern ist jederzeit die Vollendung seiner Lehre. Das Verhältnis zwischen beiden ist jederzeit durch eine Anxiety of Influence gekennzeichnet, wie Harold Bloom sie beschrieben hat; aber im Gegensatz zu Blooms Konzept der Einflussangst muss in KILL BILL nicht allein der Nachfolger Furcht vor dem >starken` Vorläufer empfinden, sondern mindestens ebenso der Lehrer vor dem allzu geschickten und ehrgeizigen Schüler.

Das abschließende Duell zwischen Bill und der Braut wird bezeichnenderweise nicht mit Schwertern, sondern mit Wörtern ausgetragen. Es geht um die Frage der Identität der Braut: Hat sie das Recht, sich für eine >bürgerliche〈 Existenz zu entscheiden, oder wäre dies für sie als »natural born killer ${ }^{26}$ nur eine oberflächliche Tarnung? Es ist kein

24 Vgl. Sigmund Freud: Totem und Tabu. Einige Übereinstimmungen im Seelenleben der Wilden und der Neurotiker [1912/13]. Frankfurt a.M.: Fischer Taschenbuch Verlag 1991, S. $195 \mathrm{f}$.

25 KILL BILL: VOLUME 2, 00:10:32.

26 KILL BILL: Volume 2, 01:42:38. 
Zufall, dass sich dieser Disput als eine Frage des Namens darstellt: Die Braut, so insistiert Bill, kann machen was sie wolle, sie bleibe stets Beatrix Kiddo, die geborene Killerin. Der Name ist in KILL BILL ein Echo des Schicksals: Der Name des Pflegers Buck korrespondiert mit dessen Lieblingstätigkeit ebenso wie der Name Bills stets nur einen Buchstaben von seinem Sterben entfernt ist. Die Braut hingegen hat vier Namen: »die Braut« (nach dem Massaker in der Kirche), »Beatrix Kiddo« (scheinbar ihr >bürgerlicher` Name, der im ersten Teil konsequent überpiept wird, im zweiten Teil jedoch genannt), »Arlene Macchiavelli« (der Name, den sie sich in El Paso selbst gibt ${ }^{27}$ ), sowie »Black Mamba« (ihr Codename im »Deadly Viper Assassination Squad«). Diesen hat offenbar Bill für sie gewählt - zumindest beschwert sich Vernita Green darüber, der Name habe weitaus eher zu ihr gepasst (»Black Mamba, I shoulda been motherfuckin Black Mamba $\ll^{28}$ ). Ihre Identität als »Black Mamba« (und vielleicht auch als »Beatrix Kiddo«) ist seine Erfindung. Das Argument Bills, die Braut habe niemals wirklich »Arlene« sein können, zielt deshalb darauf, dass sie sich niemals einen eigenen Namen geben und sich niemals von ihrem Lehrer und Mentor lösen könne. Er beharrt auf dem »Herrenrecht, Namen zu geben«, von dem Nietzsche in der Genealogie der Moral spricht ${ }^{29}$, und gerade durch die Tötung ihres Meisters erweist sich die Braut als exakt die Tötungsmaschine, die Bill unter ihrem Namen erfunden hat. Weit davon entfernt, sich von seiner Lehre zu entfernen, ist sie niemals so entschieden Bills hervorragendste Schülerin wie in dem Moment, in dem sie ihn tötet.

\section{Rache}

Das Prinzip der Rache ist ökonomisch: es beinhaltet ein Gesetz der Gabe und Gegen-Gabe. Die Rache eröffnet einen Kreislauf der Aktionen und Reaktionen, in der jede Gabe auf eine andere Gabe antwortet. Diese Antwort geschieht, wie schon das Motto des Films (»Revenge is a dish best served cold «) verrät, keineswegs augenblicklich, und ebenfalls keineswegs mit einer schlicht gleichwertigen Gegen-Gabe. Die Ökonomie der Rache fordert vielmehr eine Überbietung, eine maßlose Gegen-Gabe. Diesen Exzess der Gegen-Gabe führt in nuce die Erzählung

27 Vgl. R. Fischer: Kill Bill, S. 199.

28 KILL BILL: Volume 1, 00:12:44.

29 »Das Herrenrecht, Namen zu geben, geht so weit, dass man sich erlauben sollte, den Ursprung der Sprache selbst als Machtäusserung der Herrschenden zu fassen: sie sagen >das ist das und das <, sie siegeln jegliches Ding und Geschehen mit einem Laute ab und nehmen es dadurch gleichsam in Besitz." (Nietzsche: Zur Genealogie der Moral, Kritische Studienausgabe, Bd. 5, S. 260). 
über die Vorgeschichte Pai Meis vor, wie Bill sie der Braut am Lagerfeuer erzählt:

»Once upon a time in China, some believe around the year one-double knotthree, head priest of The White Lotus Clan, Pai Mei, was walking down the road, contemplating whatever it is that a man with Pai Mei's infinite power would contemplate - which is another way of saying: who knows -, when a Shaolin monk appeared on the road traveling in the opposite direction. As the monk and the priest crossed paths... Pai Mei - in a practically unfathomable display of generosity - gave the monk the slightest of nods. The nod was not returned. Was it the intention of the Shaolin monk to insult Pai Mei? Or did he just fail to see the generous social gesture? The motives of the monk remain unknown. What is known, were the consequences. The next morning, Pai Mei appeared at the Shaolin Temple, and demanded that the temple's head Abbot offer Pai Mei his neck, to repay the insult. The Abbot, at first, tried to console Pai Mei, only to find, Pai Mei was inconsolable. So began the massacre of the Shaolin Temple, and all sixty of the monks inside, at the fists of the White Lotus. And so began the legend of Pai Mei's Five-Point-PalmExploding-Heart-Technique. ${ }^{30}$

Seine Neigung zum Exzess lässt Pai Mei als einen literarischen Nachfahren von Kleists Michael Kohlhaas erscheinen. Wie dieser fordert Pai Mei das Prinzip der Ökonomie (den Kreislauf der Rückgabe bzw. der Erwiderung einer Gabe) ein, um es in dieser Forderung zugleich maßlos $\mathrm{zu}$ überschreiten. Bereits die Forderung nach dem Hals des Abts »to repay the insult« ist exzessiv, aber mehr noch ist es die Tötung aller Mönche des Klosters durch die legendäre >Five-Point-Palm-ExplodingHeart-Techniquer.

Das Prinzip der Rache scheint in einer Kreisbewegung darstellbar zu sein: Eine Tat antwortet auf eine vorausgehende Tat, wobei die zweite Tat die Schuld der ersten >zurückzahlt $<.{ }^{31}$ Rache scheint demnach ein sich schließender Kreis zu sein. Die allgemeinste Struktur von KILL BILL kann diesen Eindruck zunächst bestätigen: Der Schluss des zweiten Teils, in dem die Braut Bill nach einer langen Odyssee und zahllosen Gewaltakten tötet, scheint als eine Antwort auf die erste Szene des ersten Teils lesbar zu sein, in der Bill eine Kugel auf den Kopf der Braut abfeuert. Doch bereits die in die Handlung eingeschobene Vorgeschichte Pai Meis führt vor, dass Rache keineswegs als ein einfacher Zirkel

30 KiLl BiLL: Volume 2, 00:38:00.

31 Vgl. R. Fischer: Kill Bill, S. 217: "Zu den generischen Zutaten einer Rachegeschichte gehört natürlich Gewalt: Ein Gewaltakt löst den Rachefeldzug aus, eine Reihe von weiteren Gewaltakten markieren ihn bis hin zu seinem Ende, seiner Vollendung.« 
vorzustellen ist: Vielmehr löst sie die Bewegung einer ins Unendliche geöffneten Spirale aus.

Kann die Rache der Braut demnach als vollendet und gelungen bezeichnet werden? - Einerseits birgt der Akt der Rache in sich die vollständige Befriedigung eines endgültigen Abschlusses, eines Schnitts, einer Begleichung der offenen Rechnungen. So beschreibt die Braut die Tätigkeit des Rächers an einer Stelle geradezu als die Möglichkeit einer persönlichen Theodizee: »When fortune smiles on something as violent and ugly as revenge, at the time it seems proof like no other, that not only does God exist, you're doing his will. $\aleph^{32}$ Andererseits aber zeigt sich in der Tätigkeit der Rache eine irritierende >List der Vernunft $<$, in der die rächende Tötung seines Meisters als die Vollendung von dessen Lehre erscheint. Der Gott, dessen Existenz die Braut spürt und dessen Wille sie in der Rache vollzieht, ist folglich niemand anders als Bill selbst. (Noch die scheinbar willkürlichen und unkontrollierten Exzesse des Pai Mei folgen seinem Gesetz, denn es ist nur das Gesetz der Gewalt überhaupt.) Aus der Paradoxie der Traditionsverweigerung folgt demnach die Unmöglichkeit einer endgültig vollendeten Rache.

Auf der Ebene der Zeitlichkeit macht sich die Unmöglichkeit einer Vollendung der Rache bemerkbar als eine Spannung zwischen dem Diskurs der Nachahmung, der Kontinuität, der Meisterschaft und der Elternschaft einerseits und den allgegenwärtigen Gesten der Destruktion, des Abbruchs und der Diskontinuität andererseits. Wenn der Tod nach der Definition Heideggers die »Möglichkeit der schlechthinnigen Daseinsunmöglichkeit $\ll^{33}$ ist und er folglich das Nicht-Antizipierbare und Nicht-Erfahrbare schlechthin darstellt (so dass nicht einmal gesagt werden kann, was das Wort >Tod bedeutet $^{34}$ ), dann kürzt der Mörder dem anderen nicht einfach nur seine Lebenszeit ab, sondern er stößt ihn aus jeder Möglichkeit der Zeit, der Erfahrung und der Kontinuierung heraus. Der Mörder negiert die Möglichkeit des Daseins des anderen: Seine Tätigkeit stellt damit die radikalste Figur der Diskontinuierung überhaupt dar.

In KILL BILL können zahlreiche visuelle Elemente als Darstellung dieser Diskontinuierung verstanden werden. Dazu zählt auf der expliziten Ebene der Handlung vor allem die Geste des Köpfens durch ein scharfes Samuraischwert, die häufigste Tötungsart in KILL BILL, und der gesamte Kult um die besonders scharfen Schwerter des Hattori Hanzo (vgl. den Respekt, den die skalpierte O-Ren Ishii vor dem Schwert der Braut

32 KILL BILL: VOLUME 1, 00:34:29.

33 Martin Heidegger: Sein und Zeit [1927], 17. Aufl. Tübingen: Niemeyer 1993, S. 250.

34 Vgl. Jacques Derrida: Aporien. Sterben - auf die "Grenzen der Wahrheit « gefaßt sein. Übers. von Michael Wetzel. München: Fink 1998, S. 46. 
empfindet $^{35}$ ). Dazu zählt aber auch, auf der Ebene der filmischen Darstellung, die Ästhetik des schnellen Schnitts vor allem in den >action<-lastigen Szenen des ersten Teils.

Auch das Durchstreichen der fünf Namen auf der >Todesliste` der Braut kann als eine Bewegung der Diskontinuierung verstanden werden. Die Liste, auf der die Braut die Mitglieder des >Deadly Viper Assassination Squad in der Reihenfolge ihrer Tötung ausstreicht, dient zudem als eine Klammer, welche die Handlung des gesamten Films zusammenhält und auf ein stelos` ausrichtet: die Tötung Bills. Die Struktur von KILL BILL ist zwar prinzipiell seriell (es wäre ohne weiteres möglich gewesen, den Film durch weitere Namen auf der Todesliste zu verlängern), aber die finale Auseinandersetzung der Braut mit Bill verspricht eine Vollendung der Rache: die Durchbrechung der Serialität, die Beendigung von Bills Dasein und zugleich des Rachefeldzugs der Braut.

Aber die Braut vollendet durch die Tötung Bills nicht ihre Rache, sondern ihre Rolle als Schülerin Bills; darin liegt die strukturelle Ironie des Films. Der Kreislauf der Rache schließt sich nicht; die offenen Rechnungen bleiben auch nach dem Austausch unzähliger Gewalttaten offen. Insofern die Rache das Prinzip der Rache, die Ökonomie der Gewalt, nur bestätigt, ist sie ihre eigene Fortsetzung. René Girard hat diese Zirkularität als die Struktur der Rache überhaupt beschrieben:

»Die einzig befriedigende Rache angesichts vergossenen Blutes besteht darin, das Blut des Täters fließen zu lassen. Es gibt keinen eindeutigen Unterschied zwischen dem Akt, den die Rache bestraft, und der Rache selbst. Rache ist Vergeltung und ruft nach neuen Vergeltungsmaßnahmen. Das durch Rache geahndete Verbrechen versteht sich selbst nur äußerst selten als ursprüngliches Verbrechen; es will bereits Rache für ein früheres Verbrechen sein. Die Rache stellt also einen unendlichen, endlosen Prozeß dar. « ${ }^{36}$

Tatsächlich erfährt die Braut am Schluss des zweiten Teils, dass Bill sich durch das Massaker in El Paso seinerseits an ihr rächen wollte - wegen ihres vorausgehenden >Verrats $`$ an ihm. Wenn Bill dazu sagt, er habe möglicherweise überreagiert ( $\gg$ I overreacted ${ }^{37}{ }^{3}$ ), dann trifft dies auf den Rachefeldzug der Braut in gleichem Maße zu und beschreibt nichts

35 KILL BILL: Volume 1, 01:33:17.

36 René Girard: Das Heilige und die Gewalt [1972]. Übers. von Elisabeth Mainberger-Ruh. Frankfurt a.M.: Fischer Taschenbuch Verlag 1992, S. 28. Zur Anwendung der Theorie Girards auf literarische Texte vgl. exemplarisch Heiko Christians: "Mythische Reinheit und reziproke Gewalt. Lektüren zu Herman Melvilles Billy Budd. Foretopman (1891)«, in: Annette Simonis/Linda Simonis (Hg.), Mythen in Kunst und Literatur. Tradition und kulturelle Repräsentation. Köln, Weimar, Wien: Böhlau 2004, S. 318-330.

37 KILL BILL: Volume 2, 01:52:09. 
anderes als die Logik der Rache selbst. Rache ist, wie bereits Hattori Hanzo der Braut erläutert, kein gerader Weg, sondern ein Wald, in dem man sich verirren kann: »Revenge is never a straight line. It's a forest. And like in a forest, it's easy to lose your way... to get lost... to forget where you came in. ${ }^{38}$ Indem die rächende Handlung als eine direkte Fortsetzung und Nachahmung der gerächten Tat und die Rächerin als Nachfolgerin des Ermordeten dargestellt wird, erscheint Rache in KILL BILL in der Tat als ein Wald ohne Ausweg.

Dass die Logik der Nachahmung und Nachfolge auch mit dem Tod Bills nicht zu Ende geht, wird vor allem in dem kurzen Auftritt der Tochter Bills und der Braut deutlich. Die junge »B. B.« erschießt die Braut im Moment ihres Eintritts in Bills Haus im Spiel, und erzählt danach, von Bills väterlichem Stolz gedrängt, von der Ermordung ihres Goldfischs. Aus der Tochter des Killers und der Killerin kann wohl nur eine Killerin werden; auch sie wird eine Nachahmerin und Nachfolgerin ihres Vaters. Nicht zuletzt die Betrachtung ihres Lieblingsfilms SHOGUN ASSASSIN, den sie sich gemeinsam mit ihrer Mutter anschaut, bereitet sie auf ihre Zukunft vor. Der Zuschauer hört den Bericht über die Köpfung von nicht weniger als 131 Männern in diesem Film aus dem 〉Off<, während die Kamera auf die Gesichter der Braut und ihrer Tochter gerichtet ist. Die Selbstverständlichkeit, mit der Bill und die Braut ihre Tochter diesen Film sehen lassen, könnte als ironischer Beitrag zu den Debatten über >Gewalt und Medienwirkung` verstanden werden. Der Filmkonsum ist zugleich aber eine konsequente Folge aus der Philosophie der Nachfolge und Nachahmung, die das Verhalten Bills und der Braut determiniert.

Eine Fortsetzung der Gewaltakte und der Rachehandlungen ist demnach garantiert. Den Rachefeldzug der Zukunft (>KILL BILL: Volume 3r sozusagen) nimmt die Braut bereits nach der Tötung Vernita Greens vorweg, indem sie deren Tochter eine $>$ Revanche $<$ anbietet, falls sie nicht über die Ermordung ihrer Mutter hinwegkommt: »It was not my intention to do this in front of you. For that I'm sorry. But you can take my word for it, your mother had it coming. When you grow up, if you still feel raw about it, I'll be waiting. ${ }^{39}$ Die Ökonomie der Rache und die damit korrespondierende Logik der Nachfolge und Nachahmung wird kein Ende nehmen. Der Film KILL BILL erweist sich damit, bei näherer Betrachtung, nicht allein als ein Objekt kulturwissenschaftlicher Forschung, sondern ebenso als eine Reflexion über die kulturelle Logik der >offenen Rechnung<.

38 KILL BILL: VOLUME 1, 01:37:56.

39 KILL BILL: Volume 1, 00:13:50. 


\section{Literaturverzeichnis}

Bateson, Gregory: Vorstudien zu einer Theorie der Schizophrenie [1956], in: ders.: Ökologie des Geistes. Anthropologische, psychologische, biologische und epistemologische Perspektiven. Übers. Hans Günter Holl. 5. Aufl., Frankfurt a.M.: Suhrkamp 1994, S. 270301.

Bloom, Harold: Einflußangst. Eine Theorie der Dichtung, Übers. von Angelika Schweikhart, Frankfurt a.M.: Stroemfeld 1995.

Christians, Heiko: »Mythische Reinheit und reziproke Gewalt. Lektüren zu Herman Melvilles Billy Budd. Foretopman (1891)«, in: Annette Simonis/Linda Simonis (Hg.), Mythen in Kunst und Literatur. Tradition und kulturelle Repräsentation, Köln, Weimar, Wien: Böhlau 2004.

Derrida, Jacques: Aporien. Sterben - auf die »Grenzen der Wahrheit« gefaßt sein, Übers. von Michael Wetzel, München: Fink 1998.

Fischer, Robert: »Kill Bill«, in: Robert Fischer/Peter Körte/Georg Seeßlen (Hg.), Quentin Tarantino, Berlin: Bertz + Fischer 2004, S. 197-234.

Freud, Sigmund: Totem und Tabu. Einige Übereinstimmungen im Seelenleben der Wilden und der Neurotiker [1912/13]. Frankfurt a.M.: Fischer Taschenbuch Verlag 1991.

Girard, René: »Innovation und Wiederholung«, in: ders.: Die verkannte Stimme des Realen. Eine Theorie archaischer und moderner Mythen, Übers. von Petra Willim. München, Wien: Hanser 2005, S. 202-221.

Girard, René: Das Heilige und die Gewalt [1972]. Übers. von Elisabeth Mainberger-Ruh. Frankfurt a.M.: Fischer Taschenbuch Verlag 1992

Heidegger, Martin: Sein und Zeit [1927], 17. Aufl. Tübingen: Niemeyer 1993.

Kierkegaard, Søren: Furcht und Zittern. Dialektische Lyrik von Johannes de Silentio,. Übers. von Liselotte Richter, Hamburg: Europäische Verlagsanstalt 1998.

Lacoue-Labarthe, Philippe: »Hölderlin und die Griechen [1979]«, in: ders.: Die Nachahmung der Modernen. Typographien II. Übers. von Thomas Schestag. Basel, Weil am Rhein, Wien: Engeler [o.J.], S. 7185.

Lichtenberg, Georg Christoph: Sudelbücher [D 604]. - Schriften und Briefe, Bd. 1-4, Hrsg. von Wolfgang Promies, München: Hanser 1968.

Nietzsche, Friedrich: Kritische Studienausgabe in 15 Einzelbänden, Hrsg. von Giorgio Colli und Mazzino Montinari, München: Dtv; Berlin: de Gruyter 1988. 